\title{
Zentralvorstandssitzung vom 14. März 2013
}

\begin{abstract}
Arzneimittelkompendium - Vor zwei Jahren hat das Bundesverwaltungsgericht entschieden, dass Swissmedic die Zulassungsinhaberinnen nicht verpflichten kann, ihre Daten kostenpflichtig bei einem Privatanbieter zu publizieren. Das Kompendium wird somit nicht mehr veröffentlicht, und auch die Daten liegen nicht mehr in einer für die elektronischen Tools sinnvollen Struktur vor. Die FMH und die SAMW haben sich in einem Schreiben an Swissmedic gewandt und schlagen vor, die Bedürfnisse der Stakeholder gemeinsam an einem runden Tisch zu erörtern und danach Standards zu erarbeiten, welche den Aufbau einer entsprechend strukturierten Datenbank ermöglichen und zudem probate Übergangslösungen zulassen.
\end{abstract}

SwissDRG-Rechnungen - Für den Austausch von Rechnungsdaten bei DRG-Rechnungen hat man einen administrativen und einen medizinischen Datensatz festgelegt, beide Datensätze sind gleichzeitig zu übermitteln. Die Versicherer wollen nun eine «Container-Lösung» durchsetzen, welche vorsieht, die medizinischen Daten als Bestandteil der Rechnung zu schicken. Dies generiert Datenschutzprobleme und widerspricht der Verordnung, welche den Versand von verschiedenen Datensätzen vorsieht. Die Daten sind nicht separat verschlüsselt, sondern direkt lesbar und somit auch unmittelbar mit den Personenangaben verknüpft. Die FMH spricht sich klar gegen die «Container-Lösung» aus.

Health Technology Assessment (HTA) - Die Steuergruppe SwissHTA hat drei weitere Umsetzungspapiere finalisiert, welche die Konkretisierung der WZW-Kriterien, die Nutzenbewertung sowie die Wirtschaftlichkeitsbewertung beleuchten. 2013 möchte die Gruppe Konvergenzen zwischen Swiss Medical Board und SwissHTA schaffen und die me- thodischen Ansätze vergleichen. Daneben sollen die bisherigen Umsetzungspapiere gebündelt und der Schweizer HTA-Prozess weiterhin wissenschaftlich begleitet werden.

Epidemiengesetz - Die FMH hat die Revision des Epidemiengesetzes bereits 2008 befürwortet. Nachdem das Parlament die Totalrevision im vergangenen Jahr verabschiedete, wurde das Referendum ergriffen und erfolgreich realisiert. Zum Epidemiengesetz wird sich nun das Schweizer Stimmvolk äussern müssen, voraussichtlich im September 2013. Public Health Schweiz initiiert das Pro-Komitee und übernimmt den Kampagnen-Lead, die FMH tritt dem Pro-Komitee bei. Das politische Pro-Komitee formiert sich zu einem späteren Zeitpunkt.

Elektronisches Patientendossier - Das Koordinationsorgan eHealth hat bisher vor allem die technischen Voraussetzungen für ein sogenanntes dezentrales elektronisches Patientendossier spezifiziert. Nun gilt es, die Inhalte zu strukturieren. Die FMH übernimmt hierbei für den eAustrittsbericht die Federführung.

Patientenverfügung - Die Patientenverfügung, welche die FMH und die SAMW im Hinblick auf das neue Erwachsenenschutzrecht überarbeitet haben, wird sehr häufig von Privatpersonen und Organisationen im Gesundheitswesen nachgefragt. Um deren Handhabung zu erleichtern, soll nun eine Hinweiskarte zur Patientenverfügung geschaffen werden: Im Kreditkartenformat enthält sie alle notwendigen Informationen zur Person, welche die Patientenverfügung ausgefüllt hat, zum Ort, wo diese zu finden ist, und zur Vertrauensperson. Die Hinweiskarte ergänzt künftig die Patientenverfügung und ist ebenso über www.fmh.ch downloadbar.

\section{Findet Sie.}

\section{Das Ärzteverzeichnis doctorfmh.}

Dort präsent sein, wo Patienten ihren Arzt suchen. Kostenlos für Mitglieder.

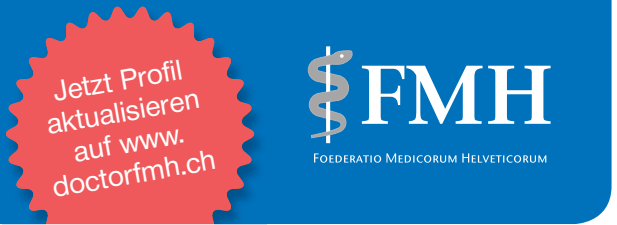

